\section{Use of intein-mediated phosphoprotein arrays to study substrate specificity of protein phosphatases}

\author{
Samvel Kochinyan, Luo Sun, Inca Ghosh, Tanya Barshevsky, Jie Xu, and \\ Ming-Qun Xu \\ New England Biolabs, Inc., Ipswich, MA, USA
}

BioTechniques 42:63-69 (January 2007)

doi $10.2144 / 000112311$

Synthetic peptides incorporating various chemical moieties, for example, phosphate groups, are convenient tools for investigating protein modification enzymes, such as protein phosphatases (PPs). However, short peptides are sometimes poor substrates, and their binding to commonly used matrices is unpredictable and variable. In general, protein substrates for PPs are superior for enzymatic assays, binding to various matrices, and Western blot analysis. The preparation and characterization of phosphoproteins, however, can be difficult and technically demanding. In this study, the intein-mediated protein ligation (IPL) technique was used to readily generate phosphorylated protein substrates by ligating a synthetic phosphopeptide to an intein-generated carrier protein $(C P)$ possessing a carboxyl-terminal thioester with a one-to-one stoichiometry. The ligated phosphoprotein (LPP) substrate was treated with a PP and subsequently subjected to array or Western blot analysis with a phosphospecific antibody. This approach is highly effective in producing arrays of protein substrates containing phosphorylated amino acid residues and has been applied for screening of PPs with specificity toward phosphorylated tyrosine, serine, or threonine residues, resulting in an approximately 240-fold increase in sensitivity in dot blot analysis compared with the use of synthetic peptides. The IPL technique overcomes the disadvantages of current methods and is a versatile system for the facile production of protein substrates containing well-defined structural motifs for the study of protein modification enzymes.

\section{INTRODUCTION}

Protein phosphorylation and dephosphorylation are predominant events in signal transduction pathways. Characterization of the specificities of protein kinases (PKs) and protein phosphatases (PPs) is pivotal for the understanding of molecular mechanisms and the determination of drug targets related to numerous diseases (1). Despite a rapidly growing appreciation of the important function of PPs in signal transduction, it is still a tremendous challenge for investigators to understand how PPs distinguish between the diversity of phosphoproteins that they encounter within the living cells. In vitro production of protein substrates for PP analysis relies on the phosphorylation of purified proteins by a PK in the presence of ATP, a reaction that is often inefficient (2-4). Although ${ }^{32} \mathrm{P}$-labeled proteins are widely used in assays of PP activity due to the high sensitivity they afford, the preparation and characterization of ${ }^{32} \mathrm{P}$-labeled substrates requires a considerable expenditure of time and effort and depends on the availability of the appropriate PK. Many proteins can also present various problems in handling, particularly with respect to solubility. Thus, it is rather difficult to obtain a sufficient quantity of phosphoprotein to use on a routine basis as a phosphatase substrate. Synthetic peptide substrates have become convenient tools for studying enzyme specificities since they are chemically well-defined entities that can be acquired in a highly purified form and in a large quantity $(5,6)$. In addition, chemical synthesis allows for the systematic alteration of the sequence and the length of the substrate peptide, which could aid in the study of substrate specificity, as well as in the optimization of an enzyme-specific substrate. However, it should be noted that in comparison to protein substrates, short phosphopeptides are sometimes not optimal enzyme substrates $(2,7)$. Enzyme-linked immunosorbent assays (ELISAs) and arrays are commonly used for peptide-based assays, whereas Western blot analysis is not suitable for small peptides. Direct synthesis of peptides on cellulose membranes (SPOT) allows for screening of large arrays of peptide substrates $(8,9)$. However, SPOT-based assays become less sensitive and nonquantitative when the amount of peptide is saturated. In ELISA, short peptides often exhibit variable binding to polystyrene, thereby causing inconsistency in signal detection. Furthermore, arraying peptides on commonly used membranes, such as low-cost nitrocellulose, is usually hindered due to low and variable binding that results in poor sensitivity and ambiguity.

We have previously demonstrated production of peptide arrays on nitrocellulose by the ligation of synthetic peptide substrates to an inteingenerated carrier protein (CP) (10) This technique, termed intein-mediated peptide array (IPA), takes advantage of the catalytic activity of an engineered intein to generate a reactive thioester at the $\mathrm{C}$ terminus of a $\mathrm{CP}$. A peptide possessing an $\mathrm{N}$-terminal cysteine residue is linked to the $\mathrm{C}$ terminus of the $\mathrm{CP}$ via a native peptide bond using intein-mediated protein ligation (IPL) (11). This method circumvents the problem associated with variable binding by small peptide substrates to various matrices, leading to successful applications for antibody characterization and epitope scanning, with an increase in sensitivity of up to $10^{4}$ fold compared with the use of peptide antigens (10).

In this report, we have constructed a diverse array of ligated phosphoprotein (LPP) substrates containing a phosphotyrosine, phosphoserine, or phosphothreonine residue. Synthetic phosphopeptides based on the phosphorylation sites of various PKs were covalently linked to the C terminus of a CP. Three types of PPs, phosphotyrosine-specific PP (PTP), phosphoserine/threonine-specific PP (PSP), and dual specificity PP (DSP), 


\section{Short Technical Reports}

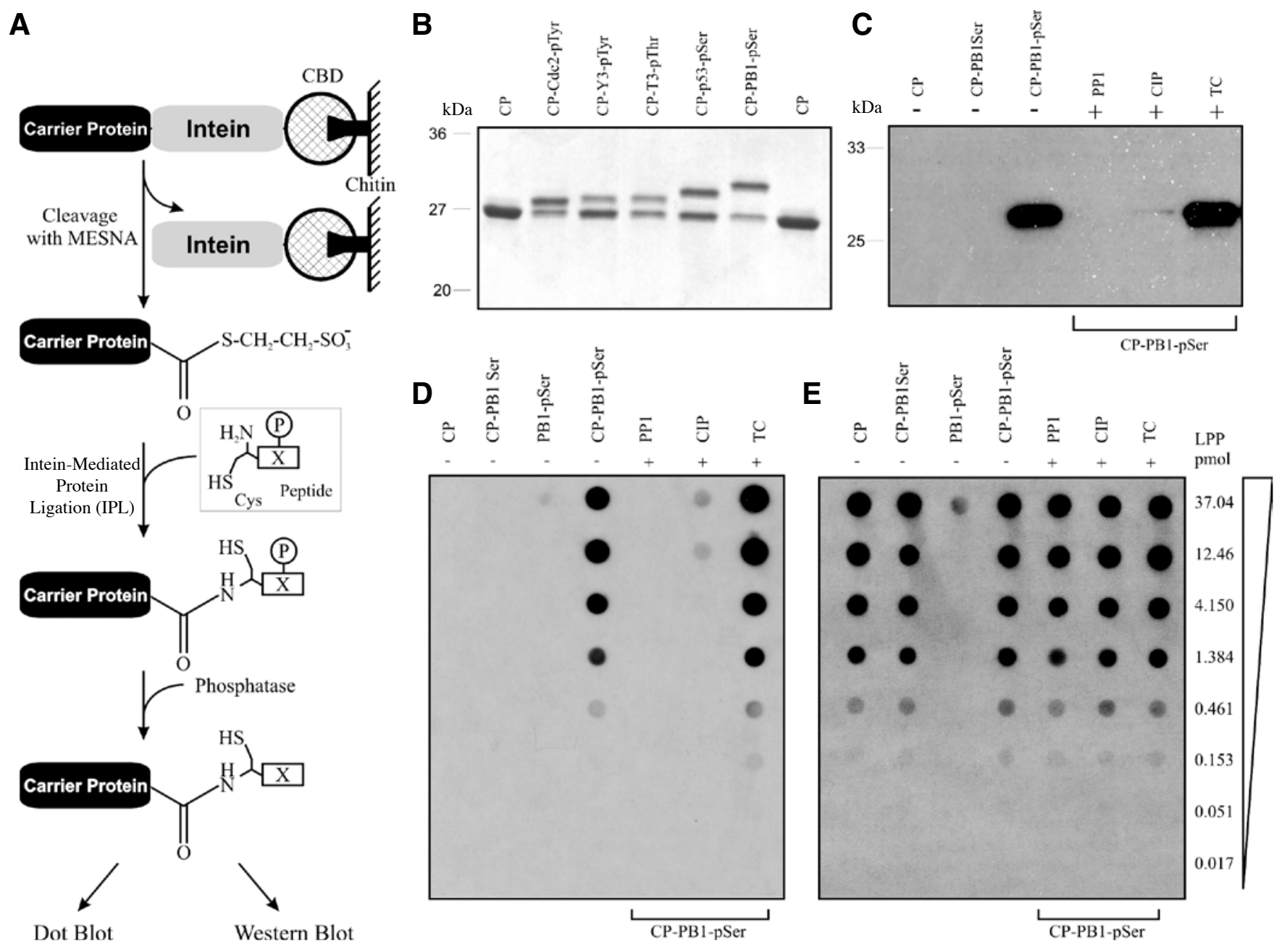

Figure 1. Production of ligated phosphoprotein substrates and evaluation of protein phosphatase (PP) assays by dot blot and Western blot analysis. (A) The production of ligated carrier protein (CP)-peptide substrates. A CP can be readily generated with a cysteine-reactive carboxyl-terminal thioester using an engineered self-splicing intein by inducing cleavage of the carrier protein-intein-chitin binding domain fusion with 2-mercaptoethanesulfonic acid (MESNA). Ligation of the $\mathrm{CP}$ to a synthetic phosphopeptide, containing an N-terminal cysteine, results in a covalent peptide bond between the two reacting molecules. The ligated phosphoprotein (LPP) serves as a substrate for PP reaction and subsequent dot blot or Western blot analysis with a phospho-specific antibody. Dephosphorylation of substrate residues in the LPP substrate yields a decrease or loss of signal. The circled P indicates a phospho group. (B) Ligation of paramyosin CP (CP, $27 \mathrm{kDa}$ ) and various phosphopeptides (Table 1) examined by sodium dodecyl sulfate polyacrylamide gel electrophoresis (SDS-PAGE). (C) Western blot analysis of PP reactions with phospho-BAD (Ser112) antibody. PB1-pSer was ligated to the CP, and the ligated substrate was treated with protein phosphatase 1 (PP1), calf intestinal phosphatase (CIP), or T cell protein tyrosine phosphatase (TC PTP). Lane 1, CP; lane 2, CP-PB1-Ser; lane 3, CP-PB1-pSer, untreated (-); lane 4, CP-PB1-pSer, treated (+) with PP1; lane 5, CP-PB1-pSer, treated with CIP; lane 6, CP-PB1-pSer, treated with TC PTP. (D) Dot blot analysis of the assay samples described above. A nonphosphopeptide, PB1-Ser, was used as a control. Column 1, CP; column 2, CP-PB1-Ser; column 3, PB1-pSer, untreated (-); column 4, CP-PB1-pSer, untreated; column 5, CP-PB1-pSer, treated (+) with PP1; column 6, CP-PB1-pSer, treated with CIP; column 7, CP-PB1pSer, treated with TC PTP. The amount of LPP substrate in each array feature is indicated on the right side. (E) The blot described in panel D was incubated with anti-CP polyclonal antibody. BAD, Bc12-antagonist of cell death.

have been used for the investigation of substrate specificity by dot blot and Western blot analysis.

\section{MATERIALS AND METHODS}

\section{Peptide Synthesis}

All peptides (Table 1) were synthesized with an $\mathrm{N}$-terminal cysteine and purified by high-performance liquid chromatography (HPLC; New England Biolabs, Ipswich, MA, USA). The phosphopeptides based on the phosphorylation sites of various PKs were described previously (10). The peptides were dissolved in 5 $\mathrm{mM}$ Tris- $\mathrm{HCl}, \mathrm{pH} 7.5,50 \mathrm{mM} \mathrm{NaCl}$ at a final concentration of $5 \mathrm{mM}$. Phosphoserine containing peptide PB1-pSer was derived from mouse
Bcl2-antagonist of cell death (BAD) (12). Phosphotyrosine-containing peptide Cdc2-pTyr15 was derived from a phosphorylation site of human cyclindependent kinase (13).

\section{Purification of the CP}

The paramyosin $\Delta$ Sal fragment (27 $\mathrm{kDa}$ ), designated as CP, from Dirofilaria immitis, was expressed as a paramyosin- 
intein-chitin binding fusion protein (PXB) and purified on chitin resin as previously described (11). Inteinmediated cleavage was carried out at $4^{\circ} \mathrm{C}$ for $16 \mathrm{~h}$ by incubation of the fusion protein bound on chitin resin in column buffer containing $20 \mathrm{mM}$ Tris- $\mathrm{HCl}, \mathrm{pH}$ $8.5,0.5 \mathrm{M} \mathrm{NaCl}$, and $50 \mathrm{mM}$ 2-mercaptoethanesulfonic acid (MESNA; Sigma, St. Louis, MO, USA). Protein concentrations of the eluted fractions were determined by Bradford assay (14).

\section{Protein Ligation}

$\mathrm{CP}(18.5 \mu \mathrm{M})$ and $1 \mathrm{mM}$ synthetic peptide were ligated overnight at $4^{\circ} \mathrm{C}$ in $100 \mathrm{mM}$ Tris- $\mathrm{HCl}, \mathrm{pH} 8.0$, and $10 \mathrm{mM}$ MESNA. The extent of ligation was evaluated by sodium dodecyl sulfate polyacrylamide gel electrophoresis (SDS-PAGE), and the ligation efficiency was typically $50 \%-75 \%$. Head-to-tail ligation of a peptide to the $\mathrm{CP}$ molecule results in a shift in mobility compared with the unligated $\mathrm{CP}$ on SDS-PAGE.

\section{PP Activity Assays}

A typical assay was conducted for 1 $\mathrm{h}$ at $30^{\circ} \mathrm{C}$ in a $10-\mu \mathrm{L}$ reaction containing approximately $7.4 \mu \mathrm{M}$ LPP substrate and $1 \mu \mathrm{L}$ phosphatase in the reaction buffer recommended by the manufacturer. Three types of PPs, PTP, PSP, and DSP, were evaluated by this study. Protein phosphatase 2A (PP2A, 500 $\mathrm{U} / \mathrm{mL}$; Promega, Madison, WI, USA) is the heterodimer of a $36-\mathrm{kDa}$ catalytic $\mathrm{C}$ subunit and a $60-\mathrm{kDa}$ A subunit obtained from human red blood cells. The other PPs were obtained from New England Biolabs. Protein phosphatase 1 (PP1, $2500 \mathrm{U} / \mathrm{mL}$ ), a PSP, is the catalytic subunit of $\alpha$-isoform of type I PP from rabbit skeletal muscle, expressed in Escherichia coli. LAR PTP (5000 U/ $\mathrm{mL}$ ) is the catalytic domain (LAR-D1) of the human receptor-like transmembrane leukocyte antigen related PTP (15). YOP protein tyrosine phosphatase (YOP, 50,000 U/mL) is the full-length product of Yersinia enterocolitica, which contains the C235R mutation (16). T cell protein tyrosine phosphatase (TC PTP, 10,000 $\mathrm{U} / \mathrm{mL}$ ) is a truncated form of human T cell PTP (TCD11) that contains the deletion of the $11-\mathrm{kDa}$ C-terminal regulatory domain from the wild-type TC PTP (17). $\lambda$ Protein phosphatase $(\lambda$-PP, 400,000 U/mL), product of bacteriophage $\lambda$, is expressed

Table 1. Peptides for Ligation and Phosphatase Assays

\begin{tabular}{|lll|}
\hline Peptide & Protein Name & Sequence \\
\hline PB1 & BAD(106-127) & CTRSRHSSYPNEYEEDEEMEEEL \\
T3 & HCDK(10-20) & CIGEGTYGVVYK \\
& IRAK(93-106) & CRARDIITAWHPPA \\
PB1-pSer & BAD(Ser112) & CTRSRHSpSYPNEYEEDEEMEEEL \\
S3-pSer & PLD1(Ser561) & CRKFSKFpSLYKQLH \\
S4-pSer & PFK-2(Ser466) & CVRMRRNpSFTPLSS \\
p53-pSer & p53(Ser15) & CSVEPPLpSQETFSDK \\
& & \\
T3-pThr & IRAK(Thr100) & CRARDIIpTAWHPPA \\
T4-pThr & DNA-PK(Thr2609) & CTPMFVEpTQASQGT \\
T5-pThr & B-Raf(Thr439) & CDRNRMKpTLGRRD \\
& & \\
Cdc2-pTyr & HCDK(Tyr15) & CIGEGTpYGVVYK \\
Y3-pTyr & PKCdelta(Tyr64) & CFDAHIpYEGRVIQ \\
Y5-pTyr & C-Kit(Tyr719) & CSDSTNEpYMDMKPG \\
Nonphosphorylated peptides and three groups of peptides containing phosphoserine, phosphothreonine \\
or phosphotyrosine residue were ligated to a thioester-tagged carrier protein (CP). The names of the pro- \\
teins from which the peptides are derived are shown in the middle column. The phosphorylated residues \\
are paired with a lowercase "p" (column on the right).
\end{tabular}

and purified from E. coli (4). Calf intestinal phosphatase (CIP, 10,000 U/mL) is a nonspecific alkaline phosphatase. For all PPs, one unit is defined as the amount of enzyme that hydrolyses 1 nmol $50 \mathrm{mM}$ p-nitrophenyl phosphate in $1 \mathrm{~min}$ at $30^{\circ} \mathrm{C}$ in a $50-\mu \mathrm{L}$ reaction. $\lambda$-PP was serially diluted in its reaction buffer prior to the titration reactions (see Figure 3). To remove unligated peptide, drop dialysis was performed using a nitrocellulose filter $(0.025 \mu \mathrm{m}$ pore size; Millipore, Billerica, MA, USA).

\section{Dot Blot Assays}

Phospho-specific antibodies (Cell Signaling Technology, Danvers, MA, USA) were used for detection of PP activity on phosphatase-treated LPP substrates by dot blots. All three phosphotyrosyl substrates (Table 1) were probed with the phosphotyrosine monoclonal antibody, P-Tyr-100. CPp53-pSer was probed with phospho-p53 monoclonal (Ser15) antibody, CP-PB1pSer with phospho-BAD monoclonal (Ser112) antibody, and CP-T3-pThr and CP-T4-pThr with phosphothreonine polyclonal antibody, P-Thr-Polyclonal. The protein blotting procedure was described previously (10). Briefly, phosphatase-treated samples were initially diluted into phosphatebuffered saline (PBS; 0.9\% $\mathrm{NaCl}, 0.2$ $\mathrm{mg} / \mathrm{mL} \mathrm{KCl}, 1.44 \mathrm{mg} / \mathrm{mL} \mathrm{Na}_{2} \mathrm{HPO}_{4}$, $0.24 \mathrm{mg} / \mathrm{mL} \mathrm{KH}_{2} \mathrm{PO}_{4}$, pH 7.4) in a microtiter plate to contain 37.04 pmols ligated protein substrate per dot $(2.666$ nmol total peptide; $5 \mu \mathrm{L}$ each reaction into $145 \mu \mathrm{L}$ PBS). Then, serial 3-fold dilutions were prepared in PBS. The $100 \mu \mathrm{L}$ diluted samples in each well were applied to a $0.45 \mu \mathrm{m}$ nitrocellulose membrane using a dot blot apparatus (Bio-Dot ${ }^{\circledR}$ Microfiltration Apparatus; Bio-Rad Laboratories, Hercules, CA, USA). The samples were drawn through the membrane by light vacuum aspiration, followed by a wash of $100 \mu \mathrm{L}$ PBS. The membrane was incubated overnight at $4^{\circ} \mathrm{C}$ in 20 $\mathrm{mL}$ diluted primary antibody (1:5000, unless specified) in Tris-buffered saline (50 mM Tris-HCl, pH 7.5, 150 $\mathrm{mM} \mathrm{NaCl}$ ) supplemented with $0.2 \%$ Tween ${ }^{\circledR} 20$ and $0.05 \%$ Triton ${ }^{\circledR}$ X-100 (TBSTT) supplemented with $2 \%$ nonfat dry milk. The membrane was washed 
with TBSTT and then incubated with diluted horseradish peroxidase (HRP)linked secondary antibody (1:5000 dilution; Cell Signaling Technology) in TBSTT with $2 \%$ nonfat dry milk for $1 \mathrm{~h}$ at room temperature, followed by three additional washes in TBSTT for 15 min each. Binding of phospho-specific antibody was detected by chemiluminescence with the Phototope ${ }^{\circledR}$-HRP Western Blot Detection System (Cell Signaling Technology). The film was digitized with Molecular Image FX (Bio-Rad Laboratories) and analyzed to determine the signal intensities with National Institutes of Health (NIH) Image J software. The signals from the array features of the untreated samples were designated as 100\% LPP substrate, converted to $0 \%$ dephosphorylation activity and compared with the signals in each row (possessing the same amount of the LPP substrate). The data was normalized by subtracting the background signal.

\section{Western Blot Analysis}

Western analysis was performed using the phospho-BAD (Ser112) antibody. Each PP-treated sample was diluted 50-fold into SDS-containing loading buffer (New England Biolabs). The samples were heated to $95^{\circ} \mathrm{C}$ for $5 \mathrm{~min}$ before an aliquot of $10 \mu \mathrm{L}$ each diluted assay sample, containing 100 ng CP, was loaded onto a $12 \%$ Trisglycine polyacrylamide gel (Invitrogen, Carlsbad, CA, USA). Proteins were resolved by SDS-PAGE. Proteins were transferred to $0.45 \mu \mathrm{m}$ nitrocellulose membrane (Schleicher \& Schuell BioScience, Keene, NH, USA) and then blocked with TBSTT supplemented with $5 \%$ nonfat dry milk. The membrane was probed with the primary antibody at 1:5000 dilution in TBSTT supplemented with $2 \%$ nonfat dry milk. After an overnight incubation at $4^{\circ} \mathrm{C}$, the membrane was washed and incubated with an anti-mouse or anti-rabbit HRP-linked immunoglobulin $\mathrm{G}(\mathrm{IgG})$ antibody $(\mathrm{H}+\mathrm{L}$; 1:5000 dilution in TBSTT with $2 \%$ dry milk) at room temperature for $1 \mathrm{~h}$. Binding of phospho-specific antibody was detected by chemiluminescence with the Phototope-HRP Western Blot Detection System.
A

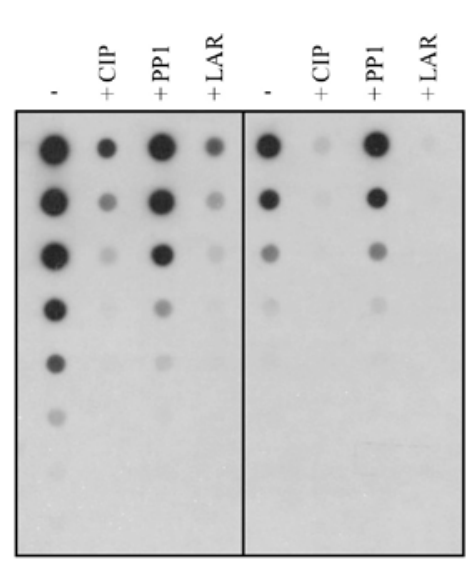

B
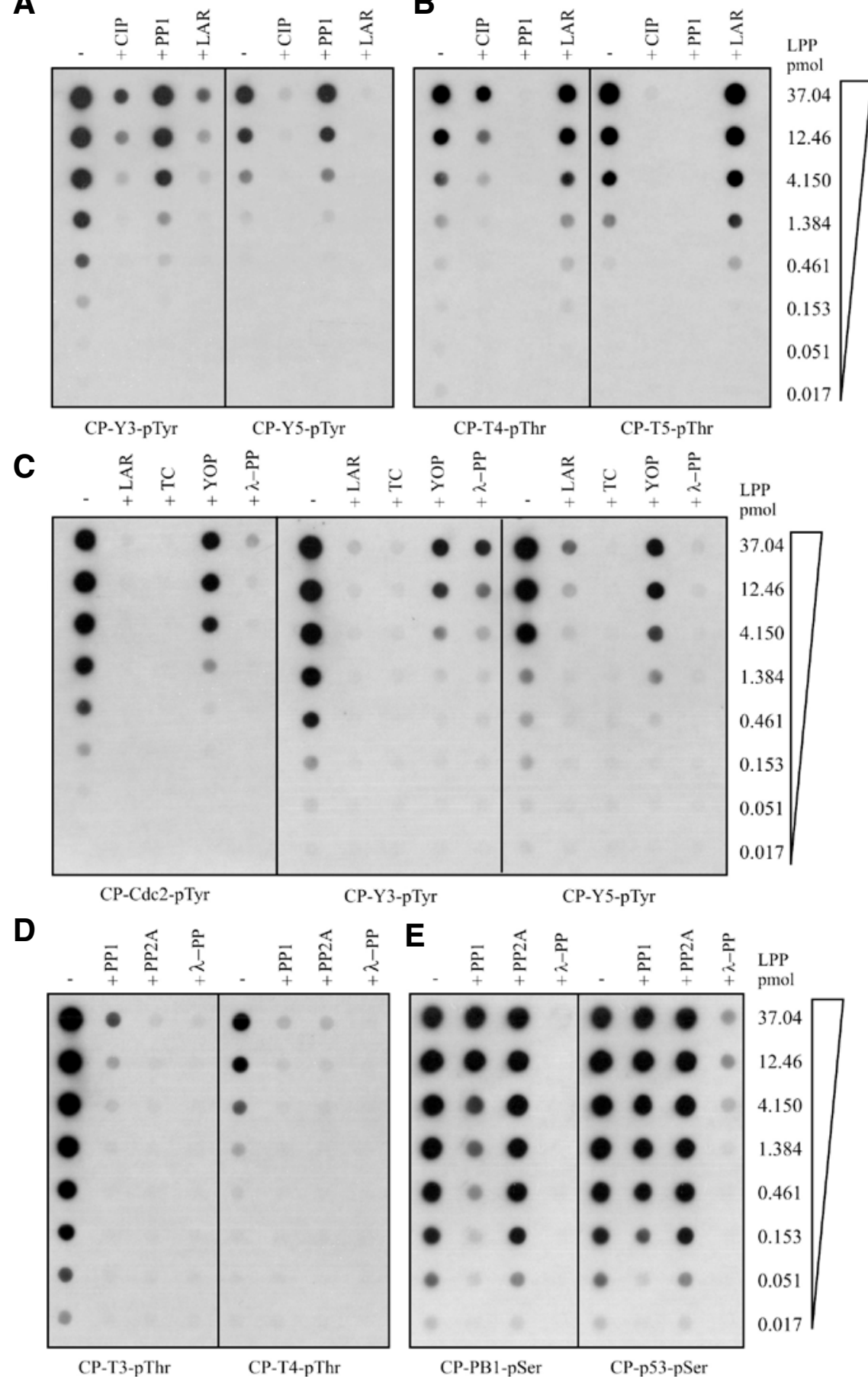

Figure 2. Analysis of protein phosphatase (PP) activity on various ligated phosphoprotein (LPP) substrates. Two different substrate groups, containing (A) a phosphotyrosine residue or (B) a phosphothreonine residue, were treated with three different phosphatases, calf intestinal phosphatase (CIP), protein phosphatase 1 (PP1), or LAR protein tyrosine phosphatase (LAR). The blot was incubated with a phosphotyrosine- or a phosphothreonine-specific antibody, depending on the substrate. (A) Columns $1-4$, untreated $(-)$ or treated (+) with CIP, PP1, or LAR; columns 5-8, untreated (-) or treated $(+)$ with CIP, PP1, or LAR. (B) Columns 1-4, untreated (-) or treated (+) with CIP, PP1, or LAR; columns 5-8, untreated (-) or treated (+) with CIP, PP1, or LAR. (C) Three phosphotyrosyl substrates, CP-Cdc2pTyr15, CP-Y3-pTyr, and CP-Y5-pTyr, were used to screen different phosphatases, LAR, YOP protein tyrosine phosphatase (YOP), T cell protein tyrosine phosphatase (TC PTP), or $\lambda$-PP. (D) LPPs containing a phosphothreonine residue were examined with a phosphothreonine-specific antibody after enzyme treatment. (E) Assays with the ligated substrates containing a phosphoserine residue were examined with a phosphoserine-specific antibody. The substrates are listed below each panel. 


\section{Short Technical Reports}

\section{RESULTS AND DISCUSSION}

To demonstrate the advantages of the IPL technique for the analysis of substrate specificity of PP, we have designed three types of peptide substrates based on the phosphorylation sites of known PKs (Table 1), containing a phosphotyrosine, phosphoserine, or phosphothreonine residue. The production of LPP substrates by IPL technique is presented in Figure $1 \mathrm{~A}$. A CP can be readily generated with a cysteine-reactive carboxyl-terminal thioester using an engineered selfsplicing intein by inducing cleavage of the CP-intein fusion with a thiol compound, MESNA. Each peptide was synthesized with capping chemistry so that only a full-length peptide possesses an amino terminal cysteine residue, necessary for IPL. Typically, ligation was conducted by mixing the $\mathrm{CP}$ to a phosphopeptide in a molar ratio of 1:50 at $4^{\circ} \mathrm{C}$, resulting in a stable peptide bond between the two reactants.

The rationale for IPA is that an intein-generated protein can function as a carrier for uniform binding of the ligated products to a matrix, thereby enhancing the sensitivity and the signal- to-noise ratio (10). We conducted our investigation using PTPs, PSPs, and a DSP. The extent of ligation was evaluated by a shift in mobility compared with the unligated $\mathrm{CP}$ on SDS-PAGE; the ligation efficiency was typically $50 \%-75 \%$ (Figure 1B). After ligating the $\mathrm{CP}$ to the phosphopeptide, the substrate was then treated with a phosphatase. The assay samples were serially diluted in PBS and blotted onto a nitrocellulose membrane under nondenaturing conditions. A phosphospecific antibody was subsequently used to detect the residual phosphorylated residue, thereby assessing the extent of dephosphorylation by the PP $(18,19)$.

A phosphoseryl peptide, PB1pSer, was ligated to a $27-\mathrm{kDa} \mathrm{CP}$, paramyosin. The ligation sample was treated with a recombinant PP1, CIP, or TC PTP. The assay samples were examined by Western blot analysis with a phosphoserine-specific antibody to detect the residual phosphoserine residue (Figure 1C). Because the linear CP-peptide conjugates migrate as a single band on SDS-PAGE, a distinct signal was detected from the ligated phosphorylated substrate. The reaction samples were also serially diluted, arrayed onto nitrocellulose, and examined with the same phosphoserine antibody used in the Western blot analysis. Use of the ligated phosphopeptide substrate allowed for the easy detection of the dephosphorylation activity of PP1 and CIP (Figure 1D). The $\mathrm{CP}$ alone yielded no signal. The treatment with PP1, a PSP, resulted in a complete loss of signal. Interestingly, CIP, a general nonspecific phosphatase (20), caused approximately 80-fold decrease in detection with the phosphoserine antibody. In contrast, TC PTP, an absolute specific PTP, showed no activity on the CP-PB1-pSer substrate. An equal amount of untreated PB1-pSer peptide was arrayed. By comparing the signals from the untreated CP-PB1-pSer array features (Figure 1D, column 4) with those from the PB1-pSer peptide array features (Figure 1D, column 3 ), the sensitivity of detection was increased by approximately 240 -fold after ligation to CP. Thus, the presence of unligated peptide made a negligible contribution to the signal. The same membrane was also subjected to examination with a rabbit polyclonal antibody raised against the $\mathrm{CP}$ (Figure 1E). The results confirmed the relatively uniform
A

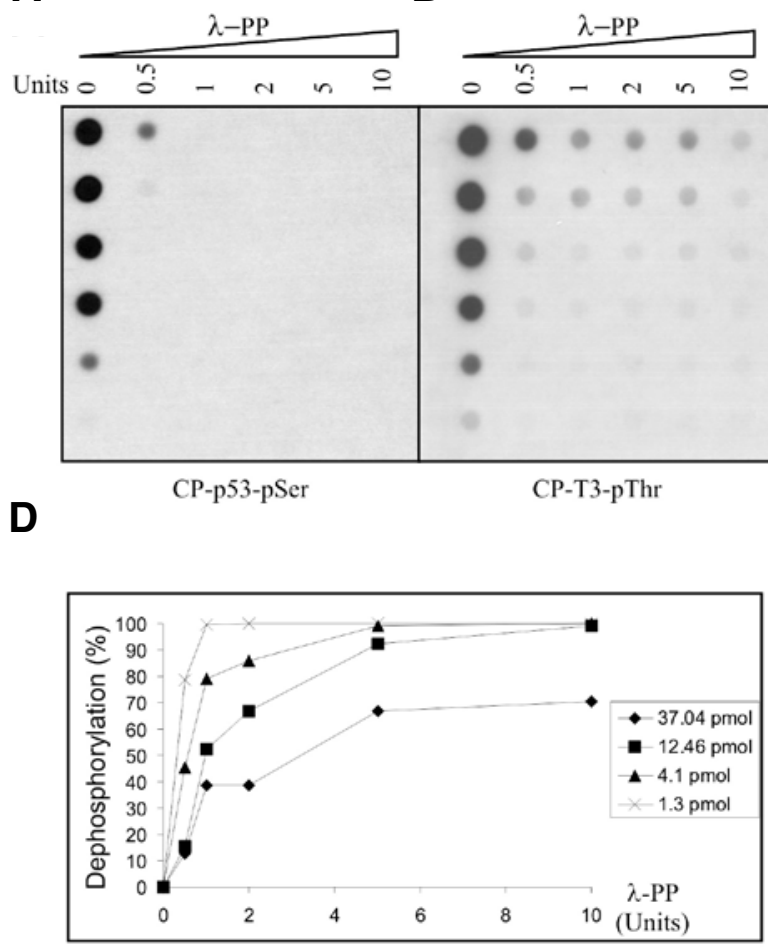

C
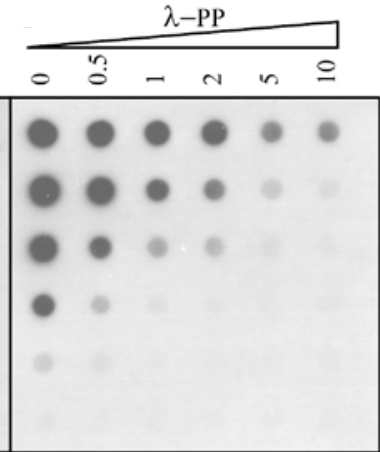

E

PP2A
CP-Cdc2-pTyr

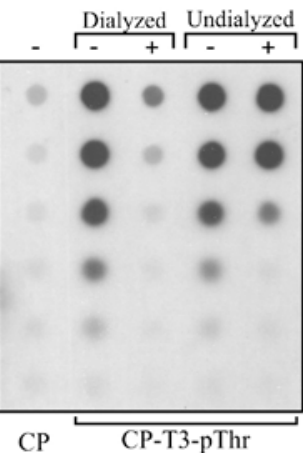

LPP

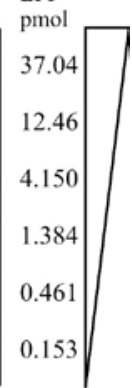
LPP 37.04
12.46
4.150
1.384
0.461
0.153

Figure 3. Enzyme titration assays by dot blot analysis. Serially diluted $\lambda$ protein phosphatase $(\lambda$-PP) was used for treatment of ligated phosphoprotein (LPP) substrates possessing (A) phosphoserine, (B) phosphothreonine, or (C) phosphotyrosine residues. Each blot was treated with a phosphospecific antibody. CP-p53-pSer was probed with phospho-p53 monoclonal (Ser15) antibody, CP-T3-pThr with a phosphothreonine polyclonal antibody, and CP-Cdc2-pTyr was treated with a phosphotyrosine monoclonal antibody. The reduced signal indicates the dephosphorylation activity of $\lambda$-PP. (D) The normalized data from scanning the blot (C) showing the dephosphorylation activity in the $\lambda$-PP titration assays. Enzyme units in a $10-\mu \mathrm{L}$ reaction are indicated. (E) Detection of protein phosphatase 2A (PP2A) activity at $0.5 \mathrm{U} / 10 \mu \mathrm{L}$ after the removal of free peptide from the ligation mixture by dialysis. The blot was treated with a phosphothreonine-specific antibody. 
application of the assay samples. The signal intensities of the array features were correlated with the intensity of the signal in the corresponding lane of the Western blot analysis. Therefore, the IPL approach makes it feasible to analyze PP assays by both dot blot and Western blot techniques.

Next, we sought to apply this approach to the analysis of PTP, PSP, and DSP using a library of phosphopeptides ligated to the $\mathrm{CP}$ (Figure 2 ). The assays were initially carried out with ligated phosphotyrosine and phosphothreonine substrates with CIP, PP1, and LAR. Both LAR (an absolute PTP) and CIP dephosphorylated the phosphotyrosine substrates, whereas PP1 appeared to have some detectable activity on the CP-Y3-pTyr substrate with an approximately 5 -fold reduction in signal intensity (Figure 2A). Since the phosphotyrosine-specific antibody exhibited variable affinity toward different phosphorylated sequences (e.g., Y3-pTyr and Y5-p-Tyr), untreated substrate was included as a control. On the other hand, LAR PTP exhibited no activity on the two phosphothreoninecontaining substrates (T4-pThr and T5pThr), but PP1 was highly active toward these substrates (Figure 2B). The PP1 assay data agrees with previous reports that recombinant PP1 retains some activity toward phosphotyrosine residues $(21,22)$. The data also indicates that CIP dephosphorylates both phosphotyrosine and phosphothreonine substrates and can function as a DSP.

A set of assays were undertaken to determine whether PPs exhibit broad specificity toward substrates. Two PTPs, LAR and TC, and a DSP, $\lambda$-PP, almost completely dephosphorylated all three phosphotyrosyl substrates, with the signal intensities reduced by approximately 27 -fold or greater (Figure 2C). YOP consistently exhibited partial dephosphorylation activity. The data confirm that these enzymes have relatively broad specificity toward phosphotyrosine substrates.

We also compared the activities by two PSPs, PP1 and PP2A, and a DSP, $\lambda$-PP, with regard to their specificity toward phosphorylated serine and threonine residues (Figure 2, D and E). All three enzymes efficiently dephosphorylated the two phosphothreonine substrates. $\lambda$-PP showed dephosphorylation of all the phosphoseryl and phosphothreonyl substrates, suggesting that $\lambda$-PP has relatively broad specificity. PP2A in this study is isolated as a catalytic dimer of a $60-\mathrm{kDa}$ A subunit and a $36-\mathrm{kDa} \mathrm{C}$ subunit, and in the absence of the B subunit, this catalytic subunit exhibits broad substrate specificity. PP2A dephosphorylated both phosphothreonine substrates but exhibited no activity on the phosphoserine substrates tested. PP1 partially dephosphorylated the ligated PB1-pSer, with approximately 27 -fold decrease in signal intensity, and only marginal activity was detected for the substrate containing p53-pSer sequence. Thus, PP1 and PP2A preferentially dephosphorylate phosphothreonyl substrates.

For all the enzymes tested, an enzyme unit is determined using a common, nonspecific substrate, p-nitrophenyl phosphate. These enzymes are supplied in a broad range of concentrations, from $500 \mathrm{U} / \mathrm{mL}$ for PP2A to $400,000 \mathrm{U} / \mathrm{mL}$ for $\lambda$-PP. Titration experiments are essential for comparing enzyme activities on the LPP substrates. As shown in Figure $3, \lambda$-PP exhibited significantly higher activity on the phosphorylated serine or threonine substrates than on the phosphotyrosine substrates. The data are in agreement with a previous report that $\lambda$-PP prefers phosphoseryl/threonyl substrates as opposed to phosphotyrosyl substrates (3). The scanned data of the spots from the dephosphorylation of CP-Cdc2-pTyr15 by $\lambda$-PP (Figure $3 \mathrm{C})$ shows the importance of enzyme titration $(0.5-1 \mathrm{U})$ and serial dilution (3- to 9-fold) of assay samples for obtaining a linear range of the signal (Figure 3D). To date, the system is not used for the measurement of a rate constant of a phosphatase. In future research, it is plausible to conduct a large-scale ligation and purification of the LPP substrate for a classical Michaelis-Menten kinetic analysis.

Although a ligation reaction containing a mixture of LPP and free peptide can be directly used for phosphatase reaction, the presence of free peptide substrate may be a problem in the interpretation of the results when an enzyme prefers a free peptide substrate. In order to detect low enzymatic activity, the removal of unligated peptide is recommended. The removal of free peptide by a drop dialysis step appeared to enhance the sensitivity in detecting the activity of diluted PP2A $(0.05 \mathrm{U}$ in a $10-\mu \mathrm{L}$ reaction; Figure $3 \mathrm{E}$ ).

Our preliminary data indicate that LPP substrates can also be applied to an on-membrane phosphatase assay, in which the LPP substrates and their serial dilutions are first arrayed onto nitrocellulose. The dot blot is subsequently treated with a phosphatase (data not shown). The on-membrane assay approach permits the investigation of specificity of an enzyme using a range of different substrates. This method, however, consumes a relatively large amount of phosphatase. Another limitation is the exclusion of untreated substrate on the same blot. In contrast, performing phosphatase treatment prior to the protein blotting step consumes less enzyme due to a small reaction volume $(10-20 \mu \mathrm{L})$ and permits comparative analysis of different phosphatases in a single assay.

In conclusion, we have developed a versatile, nonradioactive technique for conducting PP assays with ligated phosphoprotein substrates. Use of synthetic peptides and intein-generated $\mathrm{CP}$ allows for the facile production of pure protein substrates containing welldefined structural and chemical motifs for investigation of substrate specificity of PPs. Comparative analysis of three different CPs indicates that the $\mathrm{CP}$ does not appear to influence the intrinsic specificity of substrate dephosphorylation (data not shown). The CP plays the dominant role in binding to a matrix, greatly increasing sensitivity in dot blot assays. Hence, the amount of peptide in each array feature is not determined by the absolute peptide amount, but by the amount of peptide ligated to the CP molecule. Serial dilution can be performed to avoid signal saturation and to effectively screen for enzyme activity and substrate specificity. In addition, the peptide synthesis and substrate preparation is separated from array production, thereby conferring flexibility and low cost in array production. The approach provides a simple and easy platform for characterization of the increasing pool of enzymes 
involved in protein modifications and regulation of cell signaling pathways. Even though valuable information can be derived from peptide-based assays, understanding the biological roles of PPs ultimately requires investigation of their substrate specificity toward their target proteins in living cells and the biological consequences of these protein modification events.

\section{ACKNOWLEDGMENTS}

The authors thank New England Biolabs and Drs. Donald G. Comb, Thomas Evans, Jr., Christopher Noren, Elisabeth Raleigh, and Richard Roberts for their support and valuable discussion. We also thank the Organic Division for peptide synthesis.

\section{COMPETING INTERESTS STATEMENT}

The authors are employed by New England Biolabs, the manufacturer of several products discussed in this paper.

\section{REFERENCES}

1. Hardie, D.G. 2000. Protein Phosphorylation: A Practical Approach. Oxford University Press, New York.

2.Kemp, B.E. 1990. Peptides and Protein Phosphorylation. St. Vincent's Institute of Medical Research, Melbourne.

3. Slice, L.W. and S.S. Taylor. 1989. Expression of the catalytic subunit of cAMP-dependent protein kinase in Escherichia coli. J. Biol. Chem. 264:20940-20946.

4. Zhuo, S., J. Clemens, D. Hakes, D. Barford, and J. Dixon. 1993. Expression, purification, crystallization, and biochemical characterization of a recombinant protein phosphatase. J. Biol. Chem. 268:17754-17761.

5. Reimer, U., U. Reineke, and J. SchneiderMergener. 2002. Peptide arrays: from macro to micro. Curr. Opin. Biotechnol. 13:315-320.

6. Songyang, Z., K.L. Carraway III, M.J. Eck, S.C. Harrison, R.A. Feldman, M. Mohammadi, J. Schlessinger, S.R. Hubbard, et al. 1995. Catalytic specificity of protein-tyrosine kinase is critical for selective signaling. Nature 373:536-539.

7. Kennelly, P.J. and E.G. Krebs. 1991. Consensus sequences as substrate specificity determinants for protein kinases and protein phosphatases. J. Biol. Chem. 266:1555515558.

8. Frank, R. 2002. The SPOT-synthesis technique. Synthetic peptide arrays on membrane supports-principles and applications. J. Immunol. Methods 267:13-26.

9. Reineke, U., R. Volkmer-Engert, and J. Schneider-Mergener. 2001. Applications of peptide arrays prepared by the SPOT-technology. Curr. Opin. Biotechnol. 12:59-64.

10. Sun, L., J. Rush, I. Ghosh, J.R. Maunus, and M.Q. Xu. 2004. Producing peptide arrays for epitope mapping by intein-mediated protein ligation. BioTechniques 37:430-443.

11. Evans, Jr., T.C., J. Benner, and M.Q. Xu. 1998. Semisynthesis of cytotoxic proteins using a modified protein splicing element. Protein Sci. 7:2256-2264.

12. Ghosh, I., L. Sun, T.C. Evans, Jr., and M.Q. Xu. 2004. An improved method for utilization of peptide substrates for antibody characterization and enzymatic assays. J. Immunol. Methods. 293:85-95.

13. Xu, J., L. Sun, I. Ghosh, and M.Q. Xu. 2004. Western blot analysis of Src kinase assays using peptide substrates ligated to a carrier protein. BioTechniques 36:976-981.

14. Bradford, M.M. 1976. A rapid and sensitive method for the quantitation of microgram quantities of protein utilizing the principle of protein-dye binding. Anal. Biochem. 72:248254.

15. Tsujikawa, K., N. Kawakami, Y. Uchino, T. Ichijo, T. Furukawa, H. Saito, and H. Yamamoto. 2001. Distinct functions of the two protein tyrosine phosphatase domains of LAR (leukocyte common antigen-related) on tyrosine dephosphorylation of insulin receptor. Mol. Endocrinol. 15:271-280.

16. Stuckey, J.A., H.L. Schubert, E.B. Fauman, Z.Y. Zhang, J.E. Dixon, and M.A. Saper. 1994. Crystal structure of Yersinia protein tyrosine phosphatase at $2.5 \mathrm{~A}$ and the complex with tungstate. Nature 370:571-575.

17. Ruzzene, M., A. Donella-Deana, O. Marin, J.W. Perich, P. Ruzza, G. Borin, A. Calderan, and L.A. Pinna. 1993. Specificity of T-cell protein tyrosine phosphatase toward phosphorylated synthetic peptides. Eur. J. Biochem. 211:289-295.

18. Harlow, E. and D. Lane. 1988. Antibodies. CSH Laboratory Press, Cold Spring Harbor, NY.

19. Rijksen, G., B.A. van Oirschot, and G.E.J. Staal. 1991. Nonradioactive assays of protein-tyrosine kinase activity using anti-phosphotyrosine antibodies. Methods Enzymol. 200:98-107.

20. Martenson, T.M. 1984. Chemical properties, isolation, and analysis of O-phosphates in proteins. Methods Enzymol. 107:3-23.

21. Barshevsky, T. and R.J. Roberts. 1997. In vivo, in vitro, in coli. The NEB Transcript $8: 14$.

22. MacKintosh, C., A.J. Garton, A. McDonnell, D. Barford, P.T.W. Cohen, N.K. Tonks, and P. Cohen. 1996. Further evidence that inhibitor-2 acts like a chaperone to fold PP1 into its native conformation. FEBS Lett. 397:225-228.

Received 19 June 2006; accepted 25 September 2006.

Address correspondence to Ming-Qun Xu, New England Biolabs, 240 County Road, Ipswich, MA 01938-2723, USA. e-mail: xum@neb.com

To purchase reprints of this article, contact: Reprints@BioTechniques.com 